\title{
Echo Cancellation of Multipath Channel in Ultrasonic Through-steel Communication System
}

\author{
Tao Liu ${ }^{1, a}$, Xin Iv $v^{1, b}$, Qiang Guo ${ }^{1, c}$ \\ ${ }^{1}$ School of Automation \& Electrical Engineering, University of Science and Technology Beijing, \\ Beijing, 100083, China

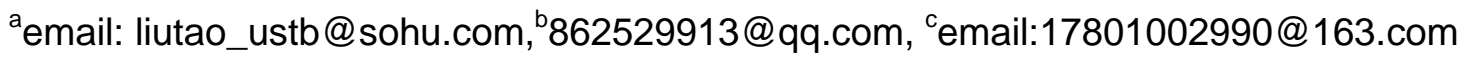

Keywords: Echo Cancellation; Multipath Channel; Through-steel Communication; Ultrasonic; Pre-Distortion

\begin{abstract}
Presence of echo is one of the challenges when using ultrasonic for transmitting data through the steel. The model of multipath channel in the ultrasonic through-steel communication system is described and the method of echo cancellation is analyzed in this paper. This method applies a pre-distortion filter to mitigate the effects of echo in order to decrease the complexity of the receiver. The method of echo cancellation is simulated in MATLAB, and simulation results show that echo is suppressed greatly comparable to the line-of-sight received signal in the ultrasonic communication system. The results show the validity.
\end{abstract}

\section{Introduction}

Ultrasound has been widely used in industry.Traditional electromagnetic wave wireless transmission cannot be applied to signal transmission through metal,the reason is that Faraday electromagnetic shielding of the metal wall has a strong inhibitory effect on electromagnetic wave transmission. Therefore, the need to transmit digital information through metal arises frequently[1,2]. The method of using ultrasonic signal as a medium to transfer data through the metal has been proved to be feasible [3].In recent years, one of the challenges of using ultrasonic communication system through metal at high rate transmission is echo received by oscilloscope, therefore, we must find a way to restrain echo and enhance the transmission rate.

Prior study about ultrasonic signal transmission data through-steel have been proved that echo can cause inter-symbol interference(ISI) in the channel[4]. The method of using pre-distortion filter to cancel echo can solve the problem of inter-symbol interference effectively [5-8].

\section{The Model of Communication System through Steel}

Shown in Fig.1, Ultrasonic communication system through steel consists of two transducer (1 MHZ non-contact transducer), rectangular steel plate and receiving apparatus of nondestructive testing pulse.

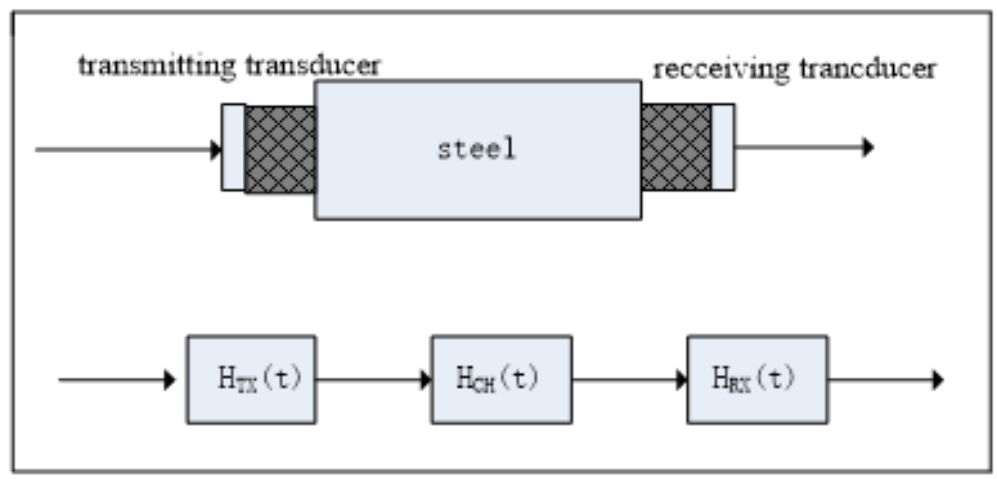

Fig. 1 The model of Ultrasonic communication system

The LOS channel impulse response is convolution of the three system impulse response[9].

$$
\mathrm{h}(\mathrm{t})=\mathrm{h}_{\mathrm{TX}}(\mathrm{t}) * \mathrm{~h}_{\mathrm{CH}}(\mathrm{t}) * \mathrm{~h}_{\mathrm{RX}}(\mathrm{t})
$$


Transducer model. Because the piezoelectric ultrasonic transducer(UT) at the center frequency resonance, its spectrum is similar to band-pass filter (BPF). In each test experiment, channel of each side use the same transducer. The transducer transfer function is as follows:

$$
\mathrm{h}_{\mathrm{T}}(\mathrm{t})=\left\{\cos \left(2 \pi f_{c} t\right)\right\} \beta e^{-2 \pi^{2}(\sigma t)^{2}}=\left\{\cos \left(2 \pi f_{c} t\right)\right\} \sqrt{2 \pi} \sigma e^{-2 \pi^{2}(\sigma t)^{2}},-\infty \mathrm{p} f \mathrm{p}+\infty, \quad \sigma \neq 0
$$

Approximation of the channel model. Isotropic medium is usually described with the basic characterization of acoustic parameters. The observed every distance attenuation is[10]:

$$
\text { Attenuation }_{\mathrm{dB}}=\frac{-20 * \log _{10}\left(\frac{x}{x_{0}}\right)}{d}, d \neq 0
$$

The function of time which Contains medium wave velocity $\mathrm{V}$ equal to the following expression:

$$
x(t)=x_{0} * e^{-\frac{\text { Attenuation }_{d B} *(t * v) * \ln (10)}{20}}
$$

If we make:

$$
\alpha=\frac{\text { Attenuation } * v * \ln (10)}{20}
$$

Equation (4) is simplified as:

$$
x(t)=x_{0} * e^{-\alpha t}
$$

Make use of the Fourier transform to convert the time domain to frequency domain:

$$
x(t)=x_{0} * e^{-\alpha t} u(t) \leftrightarrow X(f)=x_{0} \frac{1}{\alpha+j 2 \pi f}
$$

Whole LOS model approximation. The whole channel impulse response is as follows:

$$
h_{\text {channel }}(t)=\left\{K \sqrt{\pi} \sigma \cos \left(2 \pi f_{c} t\right) e^{-(\pi \sigma t)^{2}}\right\} e^{-\alpha t}=\left\{\frac{1}{1000}\left(2 \pi f_{c} t\right) e^{-(\pi \sigma t)^{2}}\right\} e^{-\alpha t}, t \geq 0 .
$$

When $t \geqslant 0$, system is a causal channel response. The channel response when excited by pulse:

$$
Y_{\text {pulse }}(t)=x_{\text {pulse }}(t) * h_{\text {channel }}(t)
$$

Received multipath signals after reflection process can be regarded as the initial dominant LOS signal and infinite time delay function:

$$
y_{\text {multipath }}(t)=y(t)+\sum_{n-1}^{\infty} y(t-n \tau)
$$

In which $\tau$ is the round trip time of acoustic signal in steel. $l$ is the length of the channel.

$$
\tau(v)=\frac{2 l}{v}
$$

For the multipath phenomenon follows the exponential decay, equation (10) converts to

$$
y_{\text {multipath }}(t)=\sum_{n=0}^{\infty} e^{-\eta t} y(t-n \tau)
$$

In which $\eta$ is the characteristics of the channel.

\section{Pre-Distortion Filter}

Fig. 2 shows The block diagram of the model[4]. In addition, $T_{1}(s)$ and $T_{2}(s)$ represent the transmit sensor and the receive sensor respectively. $\mathrm{B}(s)$ is the model of steel.

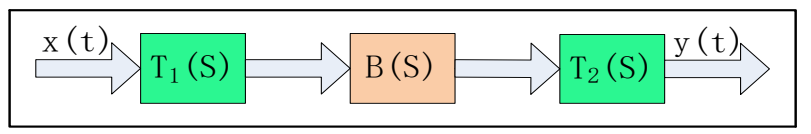

Fig.2 The block diagram of ultrasonic communication system

The design of pre-distortion filter is based on the following assumptions about two channel: each of the received echo pulses is scaled and the shifted version of the primary pulse and the amplitude of the ith echo satisfies 


$$
\operatorname{Amp}\left[e_{i+1}(t)\right]=\alpha * A m p\left[e_{i}(t)\right], 0 \mathrm{p} \alpha \mathrm{p} 1
$$

In order to build pre-distortion filter to suppress the echo the channel in Fig.2 is rearranged to Fig.3. The channel model including pre-distortion filter is shown in Fig.4.

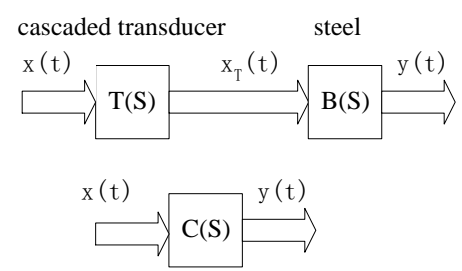

Fig. 3 the rearranged channel model

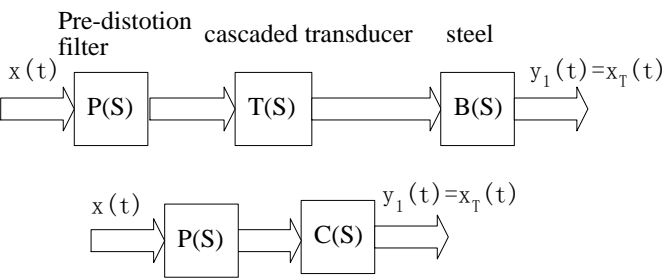

Fig. 4 the channel response including pre-distortion filter

Based the aforementioned assumption, the pulse response through steel is expressed as

$$
h_{B}(t)=\sum_{n=0}^{\infty} \delta\left(t-(1+2 n) t_{T}\right) \alpha^{1+2 n}
$$

In which $\mathrm{t}_{\mathrm{T}}$ represents the transmission time, channel attenuation constant is $\alpha(0 \mathrm{p} \alpha \mathrm{p} 1)$.The whole channel impulse response is modeled as

$$
h_{c}(t)=h_{T}(t) * h_{B}(t)=\sum_{n=0}^{\infty} h_{T}\left(t-(1+2 n) t_{T}\right) \alpha^{1+2 n}
$$

According to (14), channel impulse response of steel can be simulated as an Exponentially Weighted Moving Average filter, cascaded with a time delay. Equation (16) expresses the inverse pulse response of this filter.

$$
h_{p}(t)=\delta(t)-\alpha^{2} * \delta\left(t-2 t_{T}\right)
$$

So the channel impulse response is described as equation (17).

$$
\begin{aligned}
& h(t)=h_{p}(t)^{*} h_{c}(t)=\sum_{n=0}^{\infty} h_{T}\left(t-t_{T}-2 t_{T} n\right) \alpha^{1+2 n}-\sum_{n=0}^{\infty} h_{T}\left(t-t_{T}-2 t_{T}(1+2 n)\right) \alpha^{1+2 n} \\
& =\alpha^{*} h_{T}\left(t-t_{T}\right)+\sum_{n=1}^{\infty} h_{T}\left(t-t_{T}-2 t_{T} n\right) \alpha^{1+2 n}-\sum_{n=1}^{\infty} h_{T}\left(t-t_{T}-2 t_{T} n\right) \alpha^{1+2 n}=\alpha^{*} h_{T}\left(t-t_{T}\right)
\end{aligned}
$$

\section{Echo Cancellation}

When an acoustic wave is at normal incidence on the interface between two adjacent channel layers, each with different characteristic acoustic impedances. The energy of an acoustic signal in a channel is partitioned by each interface, with different portions of the signal traveling through the channel via many different reflective paths, each with different total acoustic lengths. This phenomenon is known as multipath signal propagation and it causes signals inserted into the channel to arrive at the channel's output as a collection of time-delayed echoes with different amplitudes and phases. When symbol rate is low, there is no need to consider the ISI because the echo from consecutive symbols sufficiently fast attenuation. However, with the increase of symbol rate, pulse arrange more closely, echo from next pulse will cause the ISI. If not corrected, it may lead to errors between symbols. The method of echo cancellation can effectively solve the problem of the ISI. Echo must be considered when the communication system is designed. Fig.5 and fig.6 are an ideal pulse and approximate channel impulse response waveforms. 


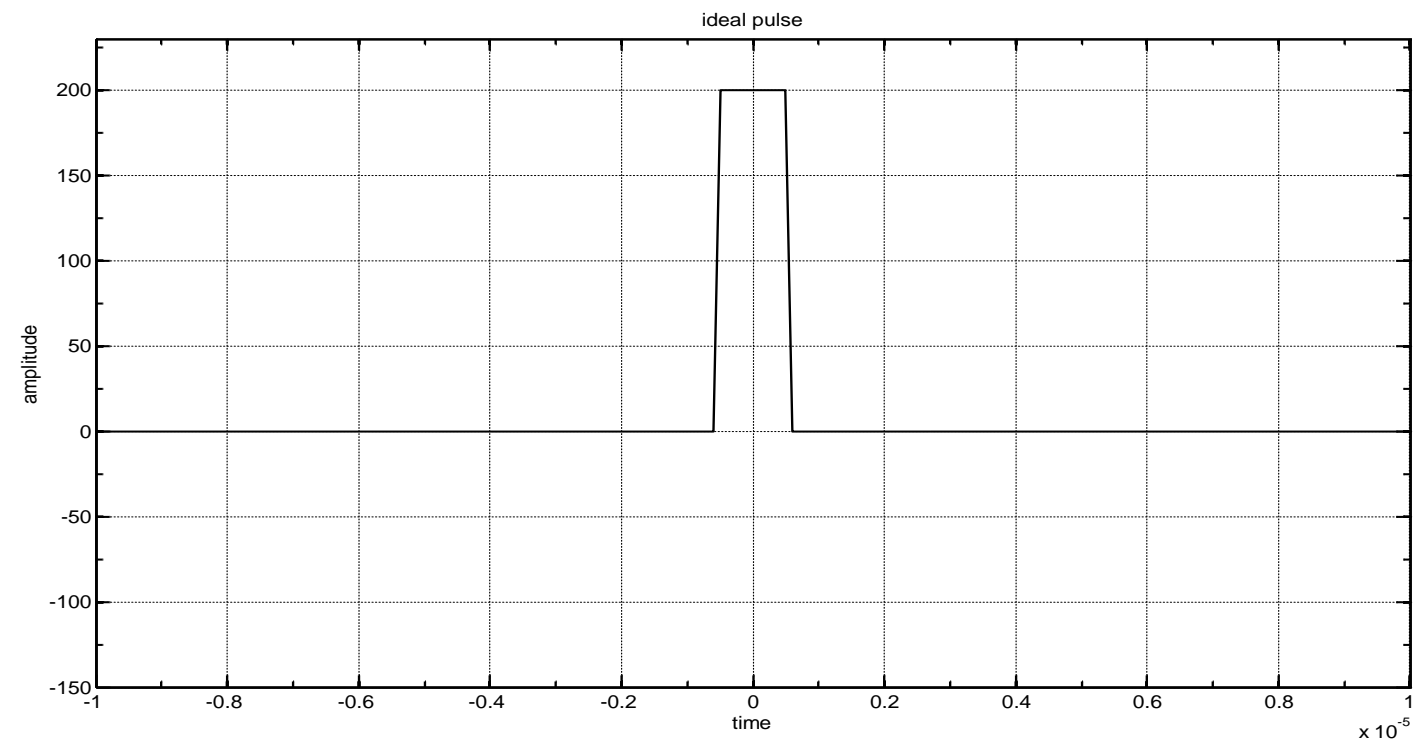

Fig. 5 Ideal pulse

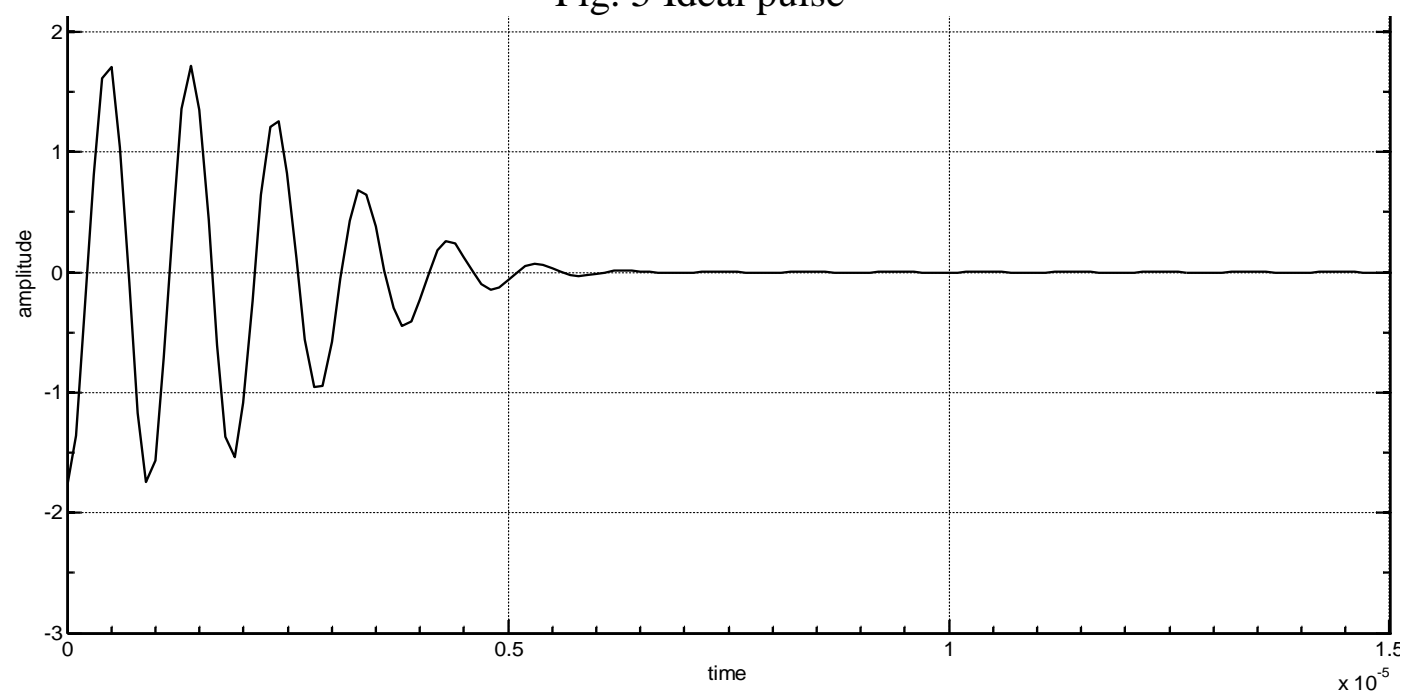

Fig. 6 channel pulse response when input Ideal pulse

Multipath channel impulse response is shown in fig.7. The transmission delay is twice of the LOS signal transmission time.

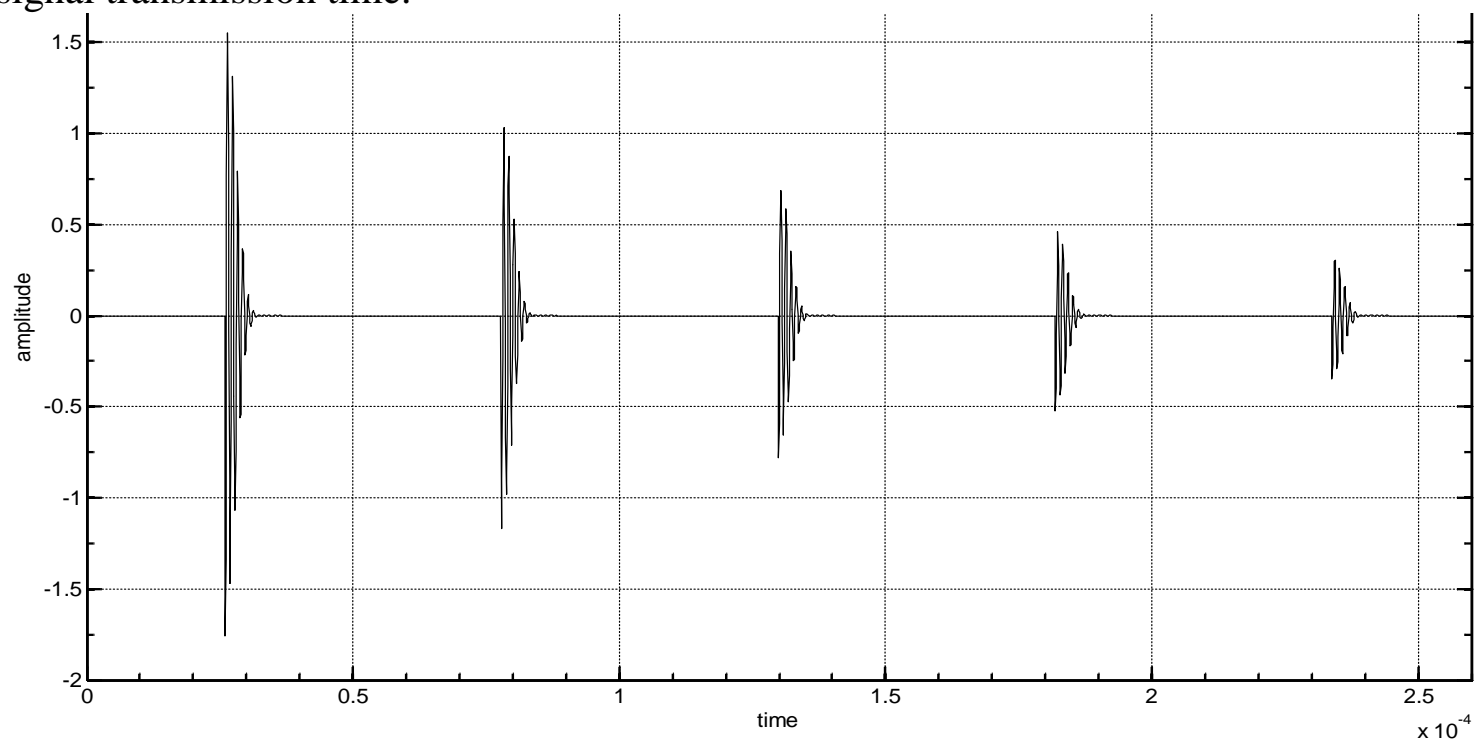

Fig.7 Multipath channel impulse response 
The echo cancellation pulse and the channel impulse response are shown in Fig.8 and Fig.9.

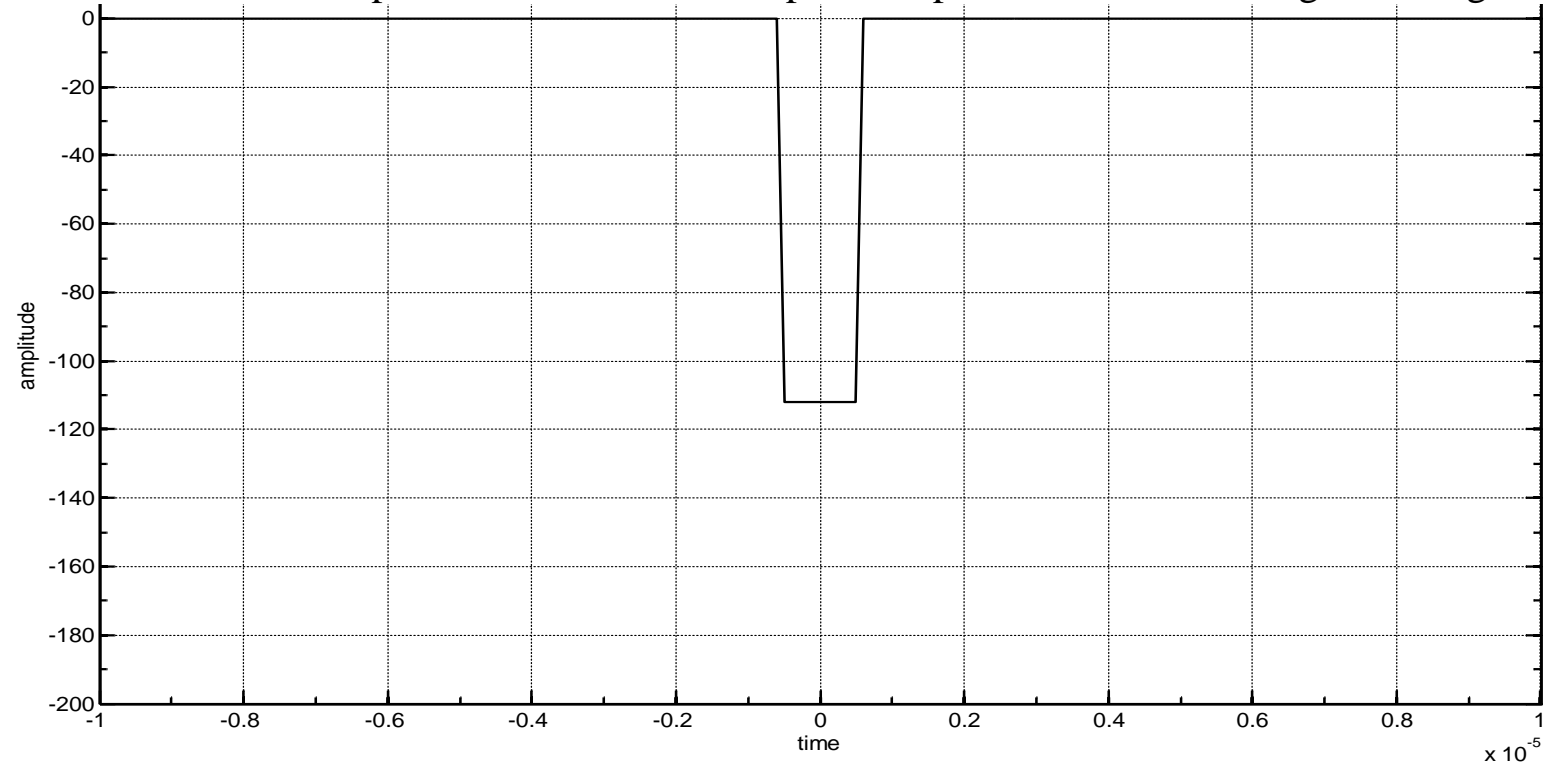

Fig. 8 Echo cancellation pulse

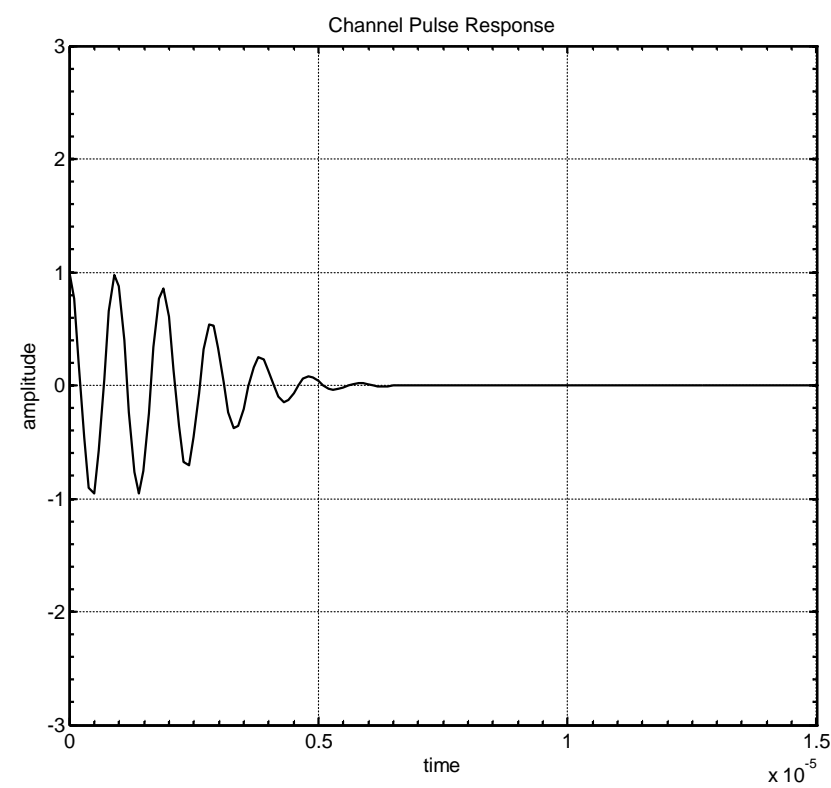

Fig. 9 channel impulse response when input echo cancellation pulse

Fig.10 shows the result when use the method of Echo cancellation. It is showed that reflection is not sufficient to reduce. Pre-distortion filter is reduced to a manageable level.

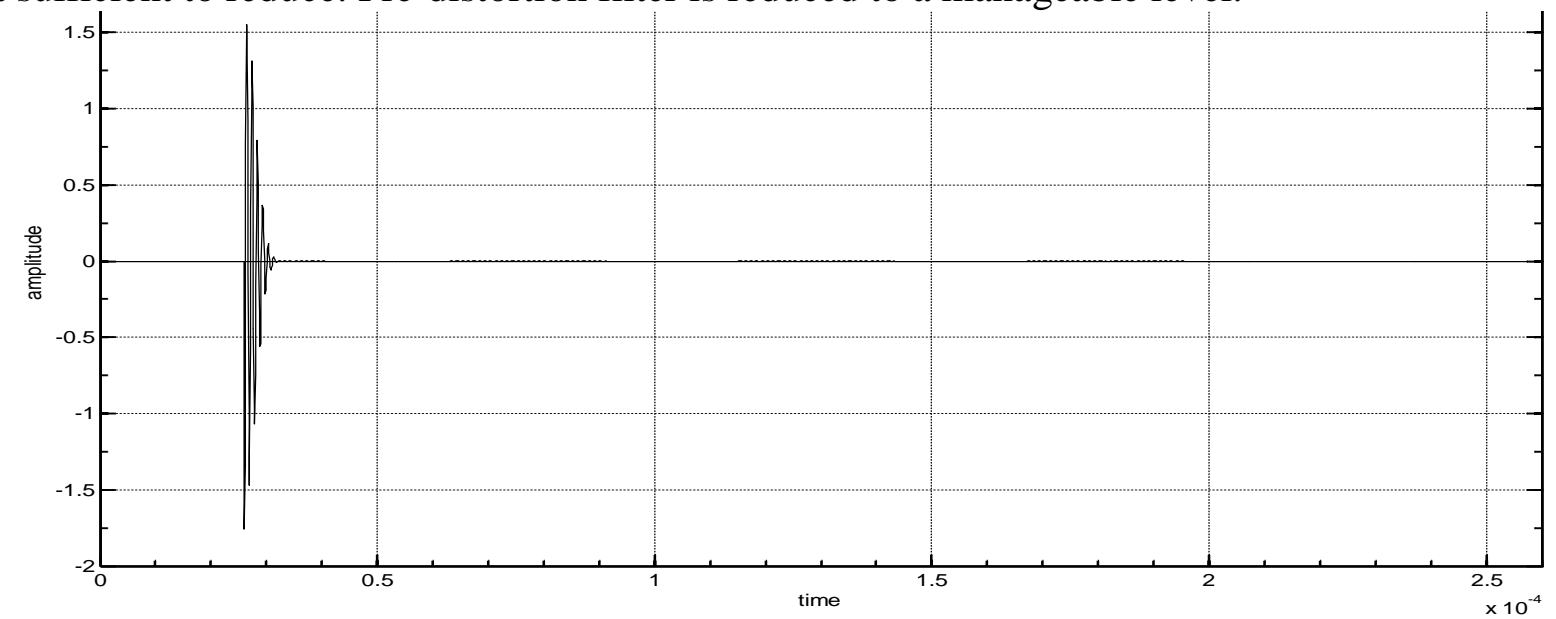

Fig. 10 channel impulse response after echo cancellation 


\section{Conclusion}

The method of multipath echo cancellation in ultrasonic wave through the steel communication system is analyzed. Simulation results show that echo cancellation algorithm reduce the echo effectively when simple channel model is used.

\section{Acknowledgement}

In this paper, the research was sponsored by the National Natural Science Foundation of China (Project No. 61372090), the Beijing Higher Education Young Elite Teacher Project (Project No. YETP0376), and the Fundamental Research Funds for the Central Universities (Project No. FRF-TP-15-059A3).

\section{References}

[1] A. Seman, M. Donnelly, and S. Mastro, Wireless systems development for distributed machinery monitoring and control, American Society of Naval Engi-neers (ASNE) Intelligent Ships Symposium VII, May 2007.

[2] C. Zacot, Shipboard wireless sensor networks utilizing zigbee technology, Master's thesis, Naval Postgraduate School, Monterey, CA, 2006.

[3] Shoudy D.A., Saulnier G.J., Scarton H.A., An Ultrasonic Through-Wall Communication System with Power Harvesting[J]. IEEE Ultrasonics Symposium 2007(1):1848-1853.

[4] R. Primerano, K. Wanuga, J. Dorn, M. Kam, and K. Dandekar, Echo cancellation for ultrasonic data transmission through a metal channel. Information Sciences and Systems, March 2007.

[5] Timothy L. Murphy, Ultrasonic Digital Communication System for a Steel Wall Multipath Channel: Method and Results. Rensselaer Polytechnic Institute, December, 2005.

[6] KONG Fan-ting.Analysis on the Technologies of Eliminate Interferences in OFDM System[J].Journal of Gansu Lianhe University(Natural Sciences).2009,23(6):34-36.

[7]WANG Han,ZHU Leiji,SHI Yusong,XING Tao,WANG Yingguan.Novel time synchronization algorithm using the cyclic prefix for OFDM systems[J].JOURNAL OF XIDIAN UNIVERSITY.2013,40(1):141-147.

[8]MA Hong-wei,LONG Hu-qiang,GUAN Yun-femg,HE Da-zhi.Single carrier frequency domain equalization for eliminating intersymbol interference[J].Research Institute of Image Communication and Information Processing,Shanghai Jiaotong University.2006(5):12-15.

[9] LAWRY T. A high performance system for wireless transmission of power and data through solid metal enclosures[D]. Ph.D. Rensselaer Polytechnic Institute. 2012.

[10] PRIMERANO R A. High bit-rate digital communication through metal channels[D]. Ph.D. Drexel University. 2010. 